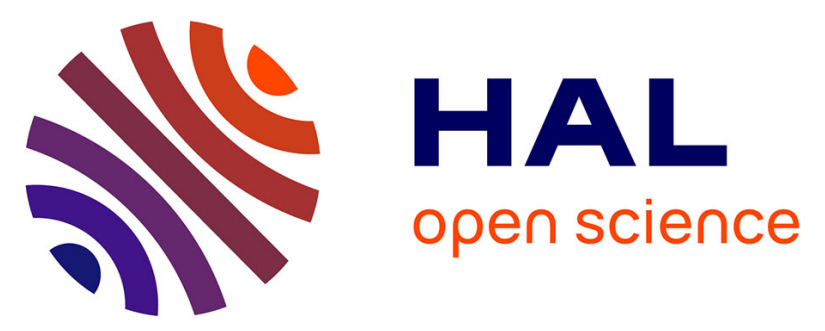

\title{
Myocardial motion and deformation patterns in an experimental swine model of acute LBBB/CRT and chronic infarct
}

Nicolas Duchateau, Marta Sitges, Adelina Doltra, Juan Fernández-Armenta, Nuria Solanes, Montserrat Rigol, Luigi Gabrielli, Etelvino Silva, Aina Barceló, Antonio Berruezo, et al.

\section{To cite this version:}

Nicolas Duchateau, Marta Sitges, Adelina Doltra, Juan Fernández-Armenta, Nuria Solanes, et al.. Myocardial motion and deformation patterns in an experimental swine model of acute LBBB/CRT and chronic infarct. International Journal of Cardiovascular Imaging, 2014, 30 (5), pp.875-887. 10.1007/s10554-014-0403-2 . hal-02282411

\section{HAL Id: hal-02282411 \\ https://hal.science/hal-02282411}

Submitted on 11 Sep 2019

HAL is a multi-disciplinary open access archive for the deposit and dissemination of scientific research documents, whether they are published or not. The documents may come from teaching and research institutions in France or abroad, or from public or private research centers.
L'archive ouverte pluridisciplinaire HAL, est destinée au dépôt et à la diffusion de documents scientifiques de niveau recherche, publiés ou non, émanant des établissements d'enseignement et de recherche français ou étrangers, des laboratoires publics ou privés. 


\title{
Myocardial motion and deformation patterns in an experimental swine model of acute LBBB/CRT and chronic infarct
}

\author{
Nicolas Duchateau $^{* 1,2,5}$, Marta Sitges ${ }^{1,2}$, Adelina Doltra ${ }^{1,2}$, Juan Fernández-Armenta ${ }^{1,2}$, Nuria Solanes ${ }^{2,1}$, Montserrat \\ Rigol $^{2,1}$, Luigi Gabrielli ${ }^{1,2,3}$, Etelvino Silva ${ }^{1,2}$, Aina Barceló ${ }^{4}$, Antonio Berruezo ${ }^{1,2}$, Lluís Mont ${ }^{1,2}$, Josep Brugada ${ }^{\dagger 1,2}$, \\ Bart Bijnens $^{\dagger 5,6}$. \\ ${ }^{1}$ Hospital Clínic, Universitat de Barcelona, c/ Villarroel 170, E-08036 Barcelona, Spain; \\ ${ }^{2}$ Institut d'investigacions Biomèdiques August Pi i Sunyer, c/ Rosselló 149-153, E-08036 Barcelona, Spain; \\ ${ }^{3}$ División de Enfermedades Cardiovasculares, Pontificia Universidad Católica de Chile, Marcoleta 367, Santiago \\ 22354 3000, Chile; \\ ${ }^{4}$ Sorin Group, Av/ de les Corts Catalanes 8, E-08173 Sant Cugat del Vallès, Barcelona, Spain; \\ ${ }^{5}$ Universitat Pompeu Fabra, c/ Roc Boronat 138, E-08018 Barcelona, Spain; \\ ${ }^{6}$ ICREA, Pg/ Lluís Companys 23, E-08010 Barcelona, Spain. \\ ${ }^{\dagger}$ The two last authors share senior authorship.
}

\author{
"Corresponding author: \\ Nicolas Duchateau \\ Arrythmia, resynchronization and cardiac imaging unit, Hospital Clínic, \\ Calle Villaroel 170, E-08036 Barcelona, Spain \\ Tel: +34.93.227.5551; Fax +34.93.451.3045 \\ Email: nicolas.duchateau@upf.edu
}




\begin{abstract}
Purpose: In cardiac resynchronization therapy (CRT), specific changes in motion/deformation happen with left-bundle-branch-block (LBBB) and following treatment. However, they remain sub-optimally studied. We propose a two-fold improvement of their characterization. This includes controlling them through an experimental model and using more suitable quantification techniques.

Methods: We used a swine model of acute LBBB and CRT with/without chronic infarct (pure-LBBB: $\mathrm{N}=11$; LBBB + left-anterior-descending infarct: $\mathrm{N}=11$ ). Myocardial displacement, velocity and strain were extracted from short-axis echocardiographic sequences using 2D speckle-tracking. The data was transformed to a single spatiotemporal system of coordinates to perform subject comparisons and quantify pattern changes at similar locations and instants.

Results: Pure-LBBB animals showed a specific intra-ventricular dyssynchrony pattern with LBBB (11/11 animals), and the recovery towards a normal pattern with CRT (10/11 animals). Pattern variability was low within the pure-LBBB population, as quantified by our method. This was not correctly assessed by more conventional measurements. Infarct presence affected the pattern distribution and CRT efficiency (improvements in 6/11 animals). Pattern changes correlated with global cardiac function (global circumferential strain) changes in all the animals (corrected: $\mathrm{p}_{\text {LBBBvsBaseline }}<0.001, \mathrm{p}_{\mathrm{CRT} \text { vBBaseline }}=\mathrm{NS}$; non-corrected: $\mathrm{p}_{\text {LBBBvsBaseline }}=\mathrm{NS}, \mathrm{p}_{\mathrm{CRTv} \text { Baseline }}=0.028$ ).

Conclusion: Our LBBB/CRT experimental model allowed controlling specific factors responsible for changes in mechanical dyssynchrony and therapy. We illustrated the importance of our quantification method to study these changes and their variability. Our findings confirm the importance of myocardial viability and of specific LBBB-related mechanical dyssynchrony patterns.
\end{abstract}

\title{
Keywords
}

Speckle-tracking; Myocardial strain; Myocardial velocity; Cardiac Resynchronization Therapy; Dyssynchrony. 


\section{Introduction}

Accurate characterization of myocardial motion and deformation patterns is essential for understanding changes in cardiac mechanics under the effect of disease and therapy [1]. Studies on Cardiac Resynchronization Therapy (CRT) mainly focused on the quantification of single value dyssynchrony indexes (e.g. time-to-event or peak measurements), and the qualitative description of the main pattern characteristics. In the first case, measurements are limited by the complexity of the mechanisms of cardiac dyssynchrony [2,3], and the difficulty of defining CRT outcome and response as a dichotomic variable [4,5]. In the second case, observations are qualitative and the pattern variability within groups of subjects is not directly measured [6-10].

In this study, we target the characterization of motion/deformation changes induced by pure left-bundlebranch-block (LBBB), and their evolution with CRT. Experimental models are relevant for such a purpose, as they allow controlling the factors responsible for such changes [11,12]. To our knowledge, only one of these models included chronic infarct [13], but tested it for lead positioning purposes. In contrast, our study partially aims at studying its influence on patterns of mechanical (dys)synchrony.

Our priority is to improve the characterization of these changes. This consists in quantifying the whole morphology and variability of motion/deformation curves (i.e., the patterns) within the studied animals. Naturally, data differs between animals in terms of the timing of physiological events, number of frames, and anatomy. For this reason, we rely on recent methods for the statistical comparison of individuals, which can be easily transposed to our data [14]. These methods derive from the fields of computational anatomy and statistical atlases, and mainly consist in aligning all the studied data to a common system of spatiotemporal coordinates.

The contribution of our study is two-fold: (i) we apply an appropriate comparison of motion and deformation patterns that overcomes the limits of previous studies on mechanical dyssynchrony; (ii) secondly, through an animal model of dyssynchrony with/without chronic infarct, we investigate the link between changes in mechanical dyssynchrony and induced electrical dyssynchrony (acute pure LBBB and following CRT). Two factors 
are tested and controlled by the experimental model: the relevance of correcting pure-LBBB dyssynchrony (noninfarcted animals), and the influence of an infarcted septum.

\section{Methods}

Animal model

Our study included 24 Landrace x Large White pigs (weight 37 [33-45] kg). Two subpopulations were created: 12 animals without structural disease, and 12 with localized chronic infarct. The infarct was induced onemonth before the LBBB induction, as a result of $90 \mathrm{~min}$ full occlusion of the left-anterior-descending coronary artery (LADi) [15]. Its position and extent were controlled 4 days before the LBBB induction using delayed-enhancement magnetic resonance (Magnetom-Symphony 1.5T, Siemens, Erlangen, Germany).

Acute LBBB was induced in all animals using radiofrequency ablation, guided through an electroanatomical mapping device (CARTO-XP, Biosense Webster, Diamond Bar, CA), so that QRS width increased $\geq 50 \%$ with respect to baseline. This resulted in $4(3-7)$ burnings, at $25 \mathrm{~mW}$ during $30 \mathrm{~s}$. This procedure is reproducible in inducing LBBB, as previously validated in [16] against other pacing protocols.

Two leads were implanted at baseline: right ventricle (RV, Beflex-RF45, Sorin Group, Milan, Italy) and left ventricle (LV, Situs-OTW, Sorin Group). The RV lead was located at the apex. The LV lead was implanted either by subxiphoid epicardial or via coronary sinus access, depending on the animal anatomy. The leads positioning was monitored by fluoroscopic guidance using a C-arm X-ray system (Arcadis Avantic, Siemens, Erlangen, Germany). Their position with respect to the infarct location was controlled a-posteriori using the electroanatomical mapping data (earliest activation locations vs. bipolar voltage maps between 0.5 and $1.5 \mathrm{mV}$ ). Pacing was performed with a pacing system analyzer (ERA-3000, Biotronik, Berlin, Germany), and optimized after the ablation. 
Further details about the leads positioning and the animal preparation protocol are given in [16]. Animal handling was approved by our Institutional Review Board and Ethics Committee, and conformed to current guidelines [17].

\section{Echocardiography}

An echocardiographic examination (Vivid-Q, GE Healthcare, Milwaukee, WI) was performed pre/postablation and during CRT using a transesophageal probe (6Tc-RS, GE Healthcare, 5MHz) via subxiphoid epicardial access. Short-axis 2D views of the LV were acquired at papillary muscles level, during breath-hold (controlled through a respiratory device). Repeatability of the imaging plane between pre-ablation, post-ablation and CRT acquisitions was checked offline using a multi-view representation. Machine settings (gain, time gain compensation, and compression) were adjusted for optimal visualization, including harmonic imaging. Sequences with insufficient image quality were discarded. Median frame rate and pixel size were of 67 [59-71] fps (heart rate: 80 [65-93] bpm) and $0.15 \times 0.15 \mathrm{~mm}^{2}$, respectively.

\section{Extraction of myocardial motion and deformation}

Speckle-tracking protocol. Myocardial motion (displacement and velocities) and deformation (strain) were estimated by 2D speckle-tracking (Echopac-v110.1.2, GE Healthcare). The temporal region of interest was manually set to one cardiac cycle with approximately $100 \mathrm{~ms}$ additional margin around it. The myocardial border was manually segmented at end-systole, as required by the software interface (delineation of the endocardium and choice of a wall thickness).

Data export and post-processing. Data was exported with the "store full trace" option, without any spatiotemporal smoothing. Motion (radial/circumferential displacement and velocity) and deformation (circumferential strain) were computed along the myocardial centerline. Drift removal was used to achieve cyclic 
motion. The position of the myocardial centerline was corrected with respect to its center-of-mass to compensate probe motion ${ }^{1}$. No additional spatial/temporal smoothing was added.

Intra- and inter-observer variability. The repeatability of the speckle-tracking procedure was evaluated on 1 non-infarcted animal pre/post-ablation, and 1 LADi animal pre-ablation. Intra-observer variability was estimated by repeating the measurements 10 times. Inter-observer variability was obtained from 3 different observers. Finally, the inter-sequence variability was evaluated by repeating the measurements on another sequence.

\section{Spatiotemporal alignment and data visualization}

Data alignment. Speckle-tracking data is not immediately comparable between subjects. Indeed, physiological events happen at different instants, and sequences also differ in number of frames and anatomy. We used the method proposed in Duchateau et al. $[14,18]$ to solve this (Figure 1). This mainly consists in aligning all the studied data to a common system of spatiotemporal coordinates. Temporal alignment was based on the matching of physiological events ${ }^{2}$. Spatial alignment consisted in transporting the motion/deformation data of each subject to a reference anatomy (the anatomy of a given animal). The importance of this spatiotemporal alignment was already discussed $[19,20]$ and is illustrated in Figures $2 a$ and $2 b$. Technical details about the whole procedure and its validation can be found in Duchateau et al. $[14,18]$. The implementation of the method was realized using Matlabv.R2007a (MathWorks, Natick, MA), and is publically available ${ }^{3}$.

Data visualization. Motion and deformation patterns are visualized by means of color-coded maps, in which the horizontal axis stands for time (one cardiac cycle), and the vertical axis stands for the position along the myocardium (Figure 1c). This visualization is similar to common commercial 2D speckle-tracking software.

\footnotetext{
${ }^{1}$ Strain is a spatially differential measure and is not affected by this correction.

2 The temporal alignment required the identification of 6 events per cycle. The onset of QRS (beginning and ending of the cycle) was identified on the ECG. Valve events (aortic/mitral valve opening/closure $[\mathrm{AVO} / \mathrm{AVC} / \mathrm{MVO} / \mathrm{MVC}]$ ) were identified using continuous-wave Doppler imaging on the corresponding valve, when possible, or visually on short-axis $2 \mathrm{D}$ views.

3 http://nicolasduchateau.wordpress.com/downloads/
} 
However, such software only offers descriptive visualization of an individual and does not allow quantitative comparison between subjects, contrary to our implementation.

\section{Pattern analysis of motion/deformation curves}

Single indexes. Differences in time-to-peak systolic strain between mid anteroseptal/inferolateral segments [21] were used as "conventional" indexes of mechanical dyssynchrony. Two types of more advanced indexes were also adapted from the ones recently proposed in Kydd et al. [22]: (i) the standard deviation of the time-to-peak circumferential strain, over the myocardial centerline ( $\left.\mathrm{STD}_{\text {time-to-peak }}\right)$, and (ii) the "wasted energy", which corresponds to the average difference between peak circumferential strain and AVC circumferential strain, over the myocardial centerline. The relevance of such measurements is commented on in the Discussion section.

Local changes in mechanical patterns. Median and first/third quartiles radial displacement, radial velocity and circumferential strain were computed at each spatiotemporal location of the color-coded maps. They were used to quantify motion/deformation changes in the non-infarcted and LADi groups.

Global changes in cardiac function. Global circumferential strain corresponded to the average of the circumferential strain maps along their vertical dimension. We used the early-diastole shortening peak of global circumferential strain (negative isovolumic relaxation peak) to estimate changes in cardiac function.

\section{Statistical analysis}

Quantitative variables were expressed as median and first/third quartiles range. Non-parametric statistical tests were used for inter-groups comparison (Mann-Whitney $U$-test) and paired data comparison (Wilcoxon signedrank test). $p$-values $<0.05$ were considered statistically significant. All data were analyzed using SPSS-v.15.0 (SPSS Inc., Chicago, IL). 


\section{Results}

\section{Animal model}

The characteristics of the studied animals are detailed in Table 1. In 22/24 animals, the successful induction of LBBB was confirmed by significant changes in the QRS morphology, axis and width (53 [46-56] ms vs. 80 [76-86] ms, $\mathrm{p}<0.001$ ). This is illustrated in Figure $3 a$ on a non-infarcted animal. The 2/24 remaining animals developed a complete atrio-ventricular block (1 non-infarcted and 1 LADi) and were discarded.

LAD infarcts ( $\mathrm{N}=11)$ were located at the anteroseptal level. More frequently affected segments were: midanteroseptal (8/11), apical-anterior (8/11), and apical-septal (10/11). Other affected segments were: mid-anterior (2/11), mid-inferoseptal (3/11), apical-inferior (2/11), apical-lateral (1/11), and apex (3/11).

\section{Intra- and inter-observer variability}

The quantitative results for the repeatability experiments are summarized in Table 2 (standard deviation of the motion/deformation curves over the whole cycle and the whole myocardium) and Figure 4 (repeated curves along the whole cycle at septal/lateral levels). Both indicate the low variability in the speckle-tracking measurements.

\section{Pattern changes: conventional assessment}

Figure $3 b$ highlights the presence after the ablation of a specific abnormal motion pattern of the septum, referred to as septal flash (SF) [6]. It consists of early-systole fast inward/outward motion, as indicated by the yellow arrows. After the ablation, this pattern was observed in all the 11 non-infarcted animals, while it was harder to assess or absent in the 11 LADi ones. With CRT, mechanical dyssynchrony was partially or totally corrected in 10 of the 11 non-infarcted animals. Similarly, improvements in synchronicity were noted for 6 of the 11 LADi animals. 
Table 1 includes measurements of mechanical dyssynchrony using time-to-peak systolic radial/circumferential strain. Large intra-population variability and no significant changes were observed with respect to pre-ablation data. This comes from the suboptimality of time-to-event indexes to characterize complex abnormal patterns $[2,3,23]$, as also illustrated in Figure 5. The wasted energy index led to similar observations. On the non-infarcted animals, significant differences were observed for the $\mathrm{STD}_{\text {time-to-peak }}$ index between pre- and postablation data, while differences between pre-ablation and CRT data were not significant. No significant differences were found for LADi animals. However, we doubt of the relevance of such a result, which is also based on time-toevent measurements.

\section{Pattern changes: group-wise spatiotemporal analysis}

Figure 6 represents the motion and deformation data for each subgroup of animals, pre/post-ablation and with CRT. This consists of median and first/third quartiles curves at septal and lateral locations, and median spatiotemporal maps.

Non-infarcted animals (Figure 6a). After the ablation, the SF is visible on the displacement and velocity data (early-systole black arrows, septal level). It is accompanied by early-systole abnormal circumferential strain (peak shortening of the septum and lateral wall stretching during the isovolumic contraction). Reduction of systolic and diastolic velocities, loss of synchronicity (absence of vertical symmetry/uniformity of the maps), and postsystolic abnormal motion (early-diastole black arrows) are also observed. With CRT, SF has disappeared, synchronicity has been restored (improved vertical symmetry/uniformity of the maps), and the contraction/relaxation patterns resemble to the pre-ablation ones.

LADi animals (Figure 6b). High variability of the circumferential strain curves is observed. This reflects the heterogeneity of infarct extent in this subgroup: fully or partially infarcted septum at the papillary muscles level vs. a more apical/anterior infarct. Motion is also slightly altered at the infarct location before the ablation. Velocities 
are lower before the ablation, in comparison with the non-infarcted animals. After the ablation, few changes are observed. No SF pattern is visible. Synchronicity is partially preserved (vertical symmetry of the maps), but motion is reduced. Post-systolic abnormal motion (early-diastole black arrows) is also observed. Few changes happen with CRT. Strain is partially improved in the lateral wall, which coincides with the non-infarcted zone.

\section{Changes in cardiac function}

Global circumferential strain values are summarized in Table 1. Non-infarcted animals showed lower global deformation after the ablation, which was almost restored with CRT. The LADi subpopulation showed a larger dispersion of global strain values before the ablation, which confirms the observations of Figure 6b. Global strain values were lower before the ablation, worsened with LBBB and were not restored with CRT.

These results are confirmed when animals are grouped according to CRT-induced improvements. Partial or total correction of LBBB-induced abnormal patterns (10 non-infarcted and 6 LADi animals) was accompanied by the restoration of cardiac function $(11.0$ [8.2-15.4] \% [pre-ablation] vs. 7.1 [5.3-8.3] \%, p<0.001 [LBBB] and 9.5 $\left.[6.3-12.5] \%, \mathrm{p}=\mathrm{NS}^{4}[\mathrm{CRT}]\right)$. On the contrary, non-correction of dyssychrony and undetermined changes (1 noninfarcted and 5 LADi animals) correlated with a lack of improvement of cardiac function (9.2 [6.8-14.5] \% [preablation] vs. $5.9[4.9-9.1] \%, \mathrm{p}=\mathrm{NS}[\mathrm{LBBB}]$ and $5.0[3.7-6.3] \%, \mathrm{p}=0.028[\mathrm{CRT}])$.

\footnotetext{
${ }^{4}$ NS: Non-significant statistical difference (p-value $>0.05$ ).
} 


\section{Discussion}

In this study, we used local motion and deformation data to quantify mechanical (dys)synchrony patterns induced by LBBB and following CRT in an animal model of dyssynchrony with/without chronic infarct. Our objectives were (i) to apply an appropriate method to compare motion and deformation patterns at each stage of the experiments; and (ii) to study the link between changes in mechanical dyssynchrony and induced electrical dyssynchrony (acute pure LBBB and following CRT).

\section{Conventional assessment of mechanical dyssynchrony}

The limits of conventional methods for the assessment of mechanical dyssynchrony have been widely debated $[24,25]$. The abundance of publications and extensively advertised multi-centric studies made the usefulness of myocardial mechanics controversial [2,3]. Single indexes such as time-to-event or single location values discard the complexity of the patterns observed (Figure 5) [23]. A similar remark can be made for more elaborated methods, such as curve analysis (e.g. cross-correlation [26] or Fourier analysis [27]) and more advanced indexes (e.g. radial discoordination $[22,28]$ or the wasted energy [22]). Note that these limitations come from the analysis that is made and not from the images. They are independent of the imaging technique, and therefore not specific to speckletracking echocardiography.

\section{Pattern analysis}

Pattern-based observations were recently reported in the context of CRT to address the problem in a more comprehensive way [6-10]. However, most works are still limited to qualitative pattern description, or single pattern-specific measurements (e.g. peak magnitude of SF, systolic rebound shortening and pre-stretch [7,8], or apical transverse motion for rocking [9]). In contrast, our method performs a more complete quantitative analysis, and includes data of multiple types (displacement, velocities and strain). Moreover, this data is studied locally all 
along the myocardial centerline and not only in predefined regions such as myocardial segments. The usefulness of the method has been demonstrated to characterize the variety of motion patterns that can be observed along the myocardium $[20,29]$.

Our approach has a second advantage for this type of studies. It allows the computation of the pattern morphology and variability within a given population. Looking at the average pattern confirms qualitative observations previously made [6-10]. Looking at its variability is essential to understand the reproducibility of the clinical observations. Both points are important for the clinical observer performing population studies. However, such a pattern analysis is only possible if data are compared at the same locations along the myocardium and the same temporal instants. With our method, data have been spatiotemporally aligned to a same reference system of coordinates. Such computations are not possible with any other existing method, and in particular with the current commercial speckle-tracking software. This relatively new pattern quantification was initially proposed in Duchateau et al. [14] and applied to the comparison of velocity patterns to those of a normal population [14,1820,29]. In the present study, we extended this framework to other parameters than velocities (displacement and strain), and illustrated it in our experimental model.

\section{Animal model}

Our method was applied to an experimental closed-chest model of pure LBBB undergoing CRT [16]. Such a model (in comparison with clinical studies) allows controlling some of the factors responsible for changes in mechanical dyssynchrony and following outcome. In particular, the non-infarcted animals before the ablation and the infarcted ones before the infarct procedure have normal cardiac function, with no antecedent of cardiac disease. This differs from patients that may show age-related physical and functional alterations. Thus, in non-infarcted animals, only the relevance of correcting LBBB-induced dyssynchrony was tested, while a second factor (infarcted septum) was tested on the LADi animals.

Previous animal studies reported the changes in dyssynchrony induced by LBBB and following CRT $[11,12]$. The closest models from our study were based on dogs. We preferred to perform on pigs for cost- 
effectiveness and experimental facilities reasons. Our observations on LBBB-related changes confirm the ones described in the literature. Our contribution is on quantitative curve analysis, and in particular on the characterization of the pattern morphology and its variability within a given population. Furthermore, to our knowledge, only one of the models mentioned in the literature included chronic infarct [13] but for lead positioning purposes, while our study examines its influence on patterns of mechanical dyssynchrony.

\section{Changes in mechanical dyssynchrony patterns}

The findings of our study confirm that a very specific pattern of mechanical dyssynchrony derives from pure LBBB. This pattern is visible on both motion and deformation data (Figures 3 and 6 ). It consists of a fast inward/outward motion of the septum during the isovolumic contraction, and early-systolic peak shortening of the septum. Stretching of the lateral wall is observed during the same temporal period. We also noticed the presence of LBBB-induced post-systolic abnormal motion, as discussed elsewhere [30]. Similar patterns were previously described [31-34], and recently referred to as septal flash [6], septal rebound stretch [7,8], or apical rocking [9]. Our experimental results confirm hypotheses about their link with electrical conduction delays and LBBB. This was recently discussed using computer simulations [7,35], experimental [11-13,36] and clinical data [7,37,38]. Further work is expected to fully understand how these specific electrical and mechanical alterations interact.

The LBBB-induced dyssynchrony patterns are observed in all the non-infarcted animals (Figure 6). However, patterns are less marked than in patients' sequences. In particular, the late systolic dyssynchrony and lateral wall stretch are sometimes subtle to assess. This may come from the electrical dyssynchrony induced on the animals. QRS width increased by more than $50 \%$, but its post-ablation duration is of $83 \pm 10 \mathrm{~ms}$, which is within the range of normal durations for human data. The use of dobutamine tests may probably increase the patterns magnitude [39], but it is not clear if the QRS duration should change. The analysis of electroanatomical data may complement this by looking for the presence of conduction block, as observed in humans [37]. One possible difference with human data may be in how ablation actually damages the left-bundle-branch (punctual burnings in pigs, against more diffuse natural damage in patients). The lack of delay between the induction of LBBB and 
imaging may be another reason for this. No time is left for remodeling processes, while remodeling may influence the mechanical dyssynchrony patterns of patients.

\section{Changes in cardiac function}

We reported that in the absence of structural disease, the correction of SF resulted in a significant improvement of cardiac function in all pigs, while the opposite was also true (the lack of SF correction in one noninfarcted animal resulted in no improvement of cardiac function). These results support previous observations on the relation between improvements of cardiac function, reverse remodeling and volume response to CRT [6,37].

\section{Local viability of the myocardium}

We also demonstrated that a localized infarct can strongly limit the presence of SF after the ablation. In case of LADi, the viability and contractility of the septum are strongly decreased at the scar location. The motion and deformation patterns of the septum are therefore altered, as previously described $[6,7,20]$. When CRT is applied, the presence of a LADi may be a possible cause of non-response, as electrical dyssynchrony is not anymore the only factor of changes in cardiac contraction.

\section{Implications}

From an image analysis/quantification point-of-view, our approach provides a complete characterization of motion and deformation patterns that is difficult to obtain with more conventional measurements. Our observations include spatiotemporal data alignment and efficient visualization tools for studying the patterns morphology and variability within a given population. This may be challenging in clinical practice, in particular with the limited access to such post-processing tools. Our approach recommends at least including qualitative pattern observations in the analysis, as performed in [6-10]. Quantitative analysis should consider comparing data at the same locations 
along the myocardium and the same physiological instants. The former may be achieved by segmental analysis, as provided in a majority of commercial speckle-tracking software. The latter is more challenging. Temporal measurements should at least be normalized by the length of the cardiac cycle, and if possible the length of the systolic/diastolic phases. We also remind that our post-processing tools are publically available ${ }^{3}$.

From a clinical point-of-view, our findings confirm the importance of the viability of the myocardium and of specific mechanical dyssynchrony patterns related to LBBB. In case of pure-LBBB and the lack of other external factors, CRT is likely to be effective, if normal motion and deformation are restored. Infarcted patients may benefit from CRT, but with a higher uncertainty due to the complexity of the infarct implications. For clinical practice, this means being critical with respect to the classical inclusion criteria for CRT, and conventional methods for assessing mechanical dyssynchrony. Pattern observations allow better understanding of the complex LBBB/CRT-induced changes, as also considered qualitatively in [6-10].

\section{Additional comments and limitations}

Our study used 2D views at the papillary muscles level. Additional 2D/3D views could improve our understanding of the patterns observed [40]. However, these views were not available in our experimental protocol with sufficient image quality and spatiotemporal resolution.

The variability of the experimental protocol is a limitation. This includes the quality of the ablation procedure (actual damage made to the left-bundle-branch), the infarct extent, and the leads location.

Finally, it should be reminded that this study uses an experimental model built for understanding purposes. No other forms of heart failure than dyssynchrony and infarction were considered (e.g. no rapid pacing was applied). Similarly, no additional factors that may influence CRT response were studied (e.g. heart failure, the lack of contractile reserve, the presence of atrial fibrillation, or a poor clinical condition at baseline). These aspects should be taken into account when looking at clinical data [20,29]. 


\section{Conclusion}

The use of a LBBB/CRT experimental model allowed controlling specific factors responsible for changes in mechanical dyssynchrony and therapy. We illustrated the importance of our quantification method, based on curve analysis rather than single measurements, to study these changes and their variability. Our study complements previous qualitative observations on specific LBBB/CRT-induced changes, aiming at a better understanding of motion and deformation in CRT candidates.

\section{Acknowledgements}

This work was partially supported by the Centre for the Development of Industrial Technology, Madrid, Spain (cvREMOD CEN-20091044) and the European Union Seventh Framework Programme (VP2HF FP7-2013611823). AD had a post-residency grant from Fundació Clínic, Barcelona, Spain. The authors acknowledge David Andreu and Santiago Sotes (Hospital Clínic, Barcelona, Spain), Daniel Traver (Biosense Webster, Barcelona, Spain), Laia Bayarri and Filippo Ziglio (Sorin Group, Barcelona/Milan, Spain/Italy) for their support on the experimental devices.

Disclosures / conflict of interest: none declared. 


\section{References}

1. Bijnens BH, Cikes M, Butakoff C, Sitges M, Crispi F (2012) Myocardial motion and deformation: What does it tell us and how does it relate to function? Fetal Diagn Ther 32:5-16. doi: 10.1159/000335649

2. Fornwalt BK, Delfino JG, Sprague WW, Oshinski JN (2009) It's time for a paradigm shift in the quantitative evaluation of left ventricular dyssynchrony. J Am Soc Echocardiogr 22:672-676. doi: 10.1016/j.echo.2009.04.012

3. Fornwalt BK (2011) The dyssynchrony in predicting response to cardiac resynchronization therapy: a call for change. J Am Soc Echocardiogr 24:180-184. doi: 10.1016/j.echo.2010.12.015

4. Fornwalt BK, Sprague WW, BeDell P, Suever JD, Gerritse B, Merlino JD, Fyfe DA, León AR, Oshinski JN (2010) Agreement is poor among current criteria used to define response to cardiac resynchronization therapy. Circulation 121:1985-1991. doi: 10.1161/CIRCULATIONAHA.109.910778

5. Cleland JG, Ghio S (2012) The determinants of clinical outcome and clinical response to CRT are not the same. Heart Fail Rev 17:755-766. doi: 10.1007/s10741-011-9268-9

6. Parsai C, Bijnens B, Sutherland GR, Baltabaeva A, Claus P, Marciniak M, Paul V, Scheffer M, Donal E, Derumeaux G, Anderson L (2009) Toward understanding response to cardiac resynchronization therapy: left ventricular dyssynchrony is only one of multiple mechanisms. Eur Heart J 30:940-949. doi: 10.1093/eurheartj/ehn481

7. Leenders GE, Lumens J, Cramer MJ, De Boeck BW, Doevendans PA, Delhaas T, Prinzen FW (2012) Septal deformation patterns delineate mechanical dyssynchrony and regional differences in contractility: analysis of patient data using a computer model. Circ Heart Fail 5:87-96. doi: 10.1161/CIRCHEARTFAILURE.111.962704

8. De Boeck BW, Teske AJ, Meine M, Leenders GE, Cramer MJ, Prinzen FW, Doevendans PA (2009) Septal rebound stretch reflects the functional substrate to cardiac resynchronization therapy and predicts volumetric and neurohormonal response. Eur J Heart Fail 11:863-871. doi: 10.1093/eurjhf/hfp107

9. Szulik M, Tillekaerts M, Vangeel V, Ganame J, Willems R, Lenarczyk R, Rademakers F, Kalarus Z, Kukulski T, Voigt JU (2010) Assessment of apical rocking: a new, integrative approach for selection of 
candidates for cardiac resynchronization therapy. Eur J Echocardiogr 11:863-869. doi: 10.1093/ejechocard/jeq081

10. Risum N, Jons C, Olsen NT, Fritz-Hansen T, Bruun NE, Hojgaard MV, Valeur N, Kronborg MB, Kisslo J, Sogaard P (2012) Simple regional strain pattern analysis to predict response to cardiac resynchronization therapy: rationale, initial results, and advantages. Am Heart J 163:697-704. doi: 10.1016/j.ahj.2012.01.025

11. Prinzen FW, Peschar M (2002) Relation between the pacing induced sequence of activation and left ventricular pump function in animals. Pacing Clin Electrophysiol 25:484-498. doi: 10.1046/j.14609592.2002.00484.x

12. Strik M, van Middendorp LB, Vernooy K (2012) Animal models of dyssynchrony. J Cardiovasc Transl Res 5:135-145. doi: 10.1007/s12265-011-9336-5

13. Rademakers LM, van Kerckhoven R, van Deursen CJ, Strik M, van Hunnik A, Kuiper M, Lampert A, Klersy C, Leyva F, Auricchio A, Maessen JG, Prinzen FW (2010) Myocardial infarction does not preclude electrical and hemodynamic benefits of cardiac resynchronization therapy in dyssynchronous canine hearts. Circ Arrhythm Electrophysiol 3:361-368. doi: 10.1161/CIRCEP.109.931865

14. Duchateau N, De Craene M, Piella G, Silva E, Doltra A, Sitges M, Bijnens BH, Frangi AF (2011) A spatiotemporal Statistical atlas of motion for the quantification of abnormal myocardial tissue velocities. Med Image Anal 15:316-328. doi: 10.1016/j.media.2010.12.006

15. Rigol M, Solanes N, Farré J, Roura S, Roqué M, Berruezo A, Bellera N, Novensà L, Tamborero D, PratVidal C, Huzman MA, Batlle M, Hoefsloot M, Sitges M, Ramírez J, Dantas AP, Merino A, Sanz G, Brugada J, Bayés-Genís A, Heras M (2010) Effects of adipose tissue-derived stem cell therapy after myocardial infarction: impact of the route of administration. J Card Fail 16:357-366. doi: 10.1016/j.cardfail.2009

16. Rigol M, Solanes N, Fernandez-Armenta J, Silva E, Doltra A, Duchateau N, Barcelo A, Gabrielli L, Bijnens B, Berruezo A, Brugada J, Sitges M (2013) Development of a Swine Model of Left Bundle Branch Block for Experimental Studies of Cardiac Resynchronization Therapy. J Cardiovasc Transl Res 6:616622. doi: $10.1007 / \mathrm{s} 12265-013-9464-1$

17. National Research Council (2011) Guide for the Care and Use of Laboratory Animals. 8th edn. The National Academies Press, Washington, DC. 
18. Duchateau N, De Craene M, Pennec X, Merino B, Sitges M, Bijnens BH (2012) Which reorientation framework for the atlas-based comparison of motion from cardiac image sequences? In: Proc. SpatioTemporal Image Analysis for Longitudinal and Time-Series Image Data, MICCAI’12 Workshop. Springer LNCS vol 7570, pp 25-37. 10.1007/978-3-642-33555-6_3

19. De Craene M, Duchateau N, Tobon-Gomez C, Ghafaryasl B, Piella G, Rhode KS, Frangi AF (2012) SPM to the heart: Mapping of 4D continuous velocities for motion abnormality quantification. In: Proc. IEEE International Symposium on Biomedical Imaging, pp 454-457. doi: 10.1109/ISBI.2012.6235582

20. Duchateau N, Doltra A, Silva E, De Craene M, Piella G, Castel MA, Mont L, Brugada J, Frangi AF, Sitges M (2012) Atlas-based quantification of myocardial motion abnormalities: added-value for the understanding the effect of cardiac resynchronization therapy. Ultrasound Med Biol 38:2186-2197. doi: 10.1016/j.ultrasmedbio.2012.08.009

21. Delgado V, Ypenburg C, van Bommel RJ, Tops LF, Mollema SA, Marsan NA, Bleeker GB, Schalij MJ, Bax JJ (2008) Assessment of left ventricular dyssynchrony by speckle tracking strain imaging comparison between longitudinal, circumferential, and radial strain in cardiac resynchronization therapy. J Am Coll Cardiol 51:1944-1952. doi: 10.1016/j.jacc.2008.02.040

22. Kydd AC, Khan FZ, O'Halloran D, Pugh PJ, Virdee MS, Dutka DP (2013) Radial strain delay based on segmental timing and strain amplitude predicts left ventricular reverse remodeling and survival after cardiac resynchronization therapy. Circ Cardiovasc Imaging 6:177-184. doi: 10.1161/CIRCIMAGING.112.000191

23. Anderson LJ, Miyazaki C, Sutherland GR, Oh JK (2008) Patient selection and echocardiographic assessment of dyssynchrony in cardiac resynchronization therapy. Circulation 117:2009-1023. doi: 10.1161/CIRCULATIONAHA.107.721332

24. Delgado V, Bax JJ (2011) Assessment of systolic dyssynchrony for cardiac resynchronization therapy is clinically useful. Circulation 123:640-655. doi: 10.1161/CIRCULATIONAHA.110.954404

25. Sung RK, Foster E (2011) Assessment of systolic dyssynchrony for cardiac resynchronization therapy is not clinically useful. Circulation 123:656-662. doi: 10.1161/CIRCULATIONAHA.110.954420

26. Silva E, Sitges M, Doltra A, Mont L, Vidal B, Castel MA, Tolosana JM, Berruezo A, González Juanatey JR, Brugada J (2010) Analysis of temporal delay in myocardial deformation throughout the cardiac cycle: 
Utility for selecting candidates for cardiac resynchronization therapy. Heart Rhythm 7:1580-1586. doi: 10.1016/j.hrthm.2010.06.034

27. Bertola B, Rondano E, Sulis M, Sarasso G, Piccinino C, Marti G, Devecchi P, Magnani A, Francalacci G, Marino PN (2009) Cardiac dyssynchrony quantitated by time-to-peak or temporal uniformity of strain at longitudinal, circumferential, and radial level: Implications for resynchronization therapy. J Am Soc Echocardiogr 22:665-671. doi: 10.1016/j.echo.2009.03.010

28. Chan YH, Kuo CT, Yeh YH, Wu LS, Wang CL, Ho WJ, Hsu LA (2013) Incremental value of radial discoordination index for the prediction of response to cardiac resynchronization therapy. Eur Heart $\mathbf{J}$ 14:213-222. doi: $10.1093 /$ ehjci/jes 112

29. Tobon-Gomez C, Duchateau N, Sebastian R, Marchesseau S, Camara O, Donal E, De Craene M, Pashaei A, Relan J, Steghofer M, Lamata P, Delingette H, Duckett S, Garreau M, Hernandez A, Rhode KS, Sermesant M, Ayache N, Leclercq C, Razavi R, Smith NP, Frangi AF (2013) Understanding the mechanisms amenable to CRT response: from pre-operative multimodal image data to patient-specific computational models. Med Biol Eng Comput 51:1235-1250. doi: 10.1007/s11517-013-1044-7

30. Ring M, Persson H, Mejhert M, Edner M (2007) Post-systolic motion in patients with heart failure - A marker of left ventricular dyssynchrony? Eur J Echocardiogr 8:352-359. doi: 10.1016/j.euje.2006.07.006

31. McDonald IG (1973) Echocardiographic demonstration of abnormal motion of the interventricular septum in left bundle branch block. Circulation 48:272-280. doi:10.1161/01.CIR.48.2.272

32. Dillon JC, Chang S, Feigenbaum H (1974) Echocardiographic manifestations of left-bundle branch-block. Circulation 49:876-880. doi: 10.1161/01.CIR.49.5.876

33. Little WC, Reeves RC, Arciniegas J, Katholi RE, Rogers EW (1982) Mechanism of abnormal interventricular septal motion during delayed left-ventricular activation. Circulation 65:1486-1491. doi: 10.1161/01.CIR.65.7.1486

34. Grines CL, Bashore TM, Boudoulas H, Olson S, Shafer P, Wooley CF (1989) Functional abnormalities in isolated left bundle branch block. The effect of interventricular asynchrony. Circulation 79:845-853. doi: 10.1161/01.CIR.79.4.845 
35. Kerckhoffs RC, Omens JH, McCulloch AD (2012) Mechanical discoordination increases continuously after the onset of left bundle branch block despite constant electrical dyssynchrony in a computational model of cardiac electromechanics and growth. Europace 14:v65-v72. doi: 10.1093/europace/eus274

36. Gjesdal O, Remme EW, Opdahl A, Skulstad H, Russell K, Kongsgaard E, Edvardsen T, Smiseth OA (2011) Mechanisms of abnormal systolic motion of the interventricular septum during left bundle-branch block. Circ Cardiovasc Imaging 4:264-273. doi: 10.1161/CIRCIMAGING.110.961417

37. Duckett SG, Camara O, Ginks MR, Bostock J, Chinchapatnam P, Sermesant M, Pashaei A, Lambiase PD, Gill JS, Carr-White GS, Frangi AF, Razavi R, Bijnens BH, Rinaldi CA (2012) Relationship between endocardial activation sequences defined by high-density mapping to early septal contraction (septal flash) in patients with left bundle branch block undergoing cardiac resynchronization therapy. Europace 14:99106. doi: 10.1093 /europace/eur235

38. Risum N, Strauss D, Sogaard P, Loring Z, Fritz-Hansen T, Bruun NE, Wagner G, Kisslo J (2013) Left bundle-branch block: The relationship between electrocardiogram electrical activation and echocardiography mechanical contraction. Am Heart J 166:340-348. doi: 10.1016/j.ahj.2013.04.005

39. Parsai C, Baltabaeva A, Anderson L, Chaparro M, Bijnens BH, Sutherland GR (2009) Low-dose dobutamine stress echo to quantify the degree of remodelling after cardiac resynchronization therapy. Eur Heart J 30:950-958. doi: 10.1093/eurheartj/ehp050

40. Duchateau N, Bijnens BH, D'hooge J, Sitges M (2013) Three-dimensional assessment of cardiac motion and deformation. In: Shiota T (ed) 3D echocardiography, 2nd edn. CRC Press, pp 201-213 
Table 1. Animal characteristics and single motion/deformation measurements.

\begin{tabular}{|c|c|c|c|c|c|c|}
\hline & \multicolumn{3}{|c|}{ Non-infarcted $(\mathrm{N}=11)$} & \multicolumn{3}{|c|}{ LAD infarcted $(\mathrm{N}=11)$} \\
\hline & Pre-ablation & LBBB & CRT & Pre-ablation & LBBB & CRT \\
\hline Weight (kg) & & $35[30 / 36]$ & & & $45[38 / 55]$ & \\
\hline \multirow[t]{2}{*}{ QRS width (ms) } & $55[50 / 57]$ & $84[78 / 88]$ & . & $52[45 / 55]$ & $78[75 / 86]$ & . \\
\hline & & $(\mathrm{p}=0.005)^{*}$ & & & $(\mathrm{p}=0.005)^{*}$ & \\
\hline $\begin{array}{lll}\text { Radial strain delay } & \end{array}$ & $0[0 / 0]$ & $0[-15 / 29]$ & $0[-38 / 14]$ & $0[-25 / 0]$ & $0[-99 / 17]$ & $0[-105 / 16]$ \\
\hline (ms) & & $(\mathrm{p}=\mathrm{NS})^{*}$ & $(\mathrm{p}=\mathrm{NS})^{+}$ & & $(\mathrm{p}=\mathrm{NS})^{*}$ & $(\mathrm{p}=\mathrm{NS})^{+}$ \\
\hline Circumferential strain & $22[-42 / 51]$ & $47[-103 / 101]$ & $49[-38 / 91]$ & $-28[-51 / 0]$ & $-71[-177 / 98]$ & $-43[-102 / 63]$ \\
\hline delay (ms) & & $(\mathrm{p}=\mathrm{NS})^{*}$ & $(\mathrm{p}=\mathrm{NS})^{+}$ & & $(\mathrm{p}=\mathrm{NS})^{*}$ & $(\mathrm{p}=\mathrm{NS})^{+}$ \\
\hline STD $_{\text {time-to-peak }}$ & $15[14 / 19]$ & $22[20 / 26]$ & $20[10 / 22]$ & $17[14 / 23]$ & $19[13 / 23]$ & $20[14 / 25]$ \\
\hline ( $\%$ of cycle) & & $(\mathrm{p}=0.006)^{*}$ & $(\mathrm{p}=\mathrm{NS})^{+}$ & & $(\mathrm{p}=\mathrm{NS})^{*}$ & $(\mathrm{p}=\mathrm{NS})^{+}$ \\
\hline \multirow[t]{2}{*}{ Wasted energy (\%) } & $6.3[5.3 / 7.6]$ & $7.6[7.1 / 8.3]$ & $5.9[3.4 / 9.2]$ & $5.6[3.4 / 11.3]$ & $6.1[4.3 / 10.0]$ & $8.9[5.0 / 12.0]$ \\
\hline & & $(\mathrm{p}=\mathrm{NS})^{*}$ & $(\mathrm{p}=\mathrm{NS})^{+}$ & & $(\mathrm{p}=\mathrm{NS})^{*}$ & $(p=N S)^{+}$ \\
\hline \multirow[t]{2}{*}{ Global strain (\%) } & $11.3[9.6 / 14.6]$ & $6.7[6.0 / 8.1]$ & $8.9[6.9 / 12.8]$ & $8.2[6.4 / 15.6]$ & $5.9[4.7 / 11.7]$ & $5.9[3.9 / 11.0]$ \\
\hline & & $(\mathrm{p}=0.003)^{*}$ & $(\mathrm{p}=\mathrm{NS})^{+}$ & & $(\mathrm{p}=0.01)^{*}$ & $(\mathrm{p}=0.01)^{+}$ \\
\hline
\end{tabular}

LAD: left-anterior-descending. LBBB: left-bundle-branch-block. CRT: cardiac resynchronization therapy.

NS: Non-significant statistical difference (p-value $>0.05)$

*: Wilcoxon signed-rank test (LBBB vs. Pre-ablation)

${ }^{+}$: Wilcoxon signed-rank test (CRT vs. Pre-ablation) 
Table 2. Variability of the speckle-tracking procedure. Intra-observer, inter-observer, and inter-sequence variability (standard deviation of the motion/deformation curves over the whole cycle and the myocardial centerline).

\begin{tabular}{lcccc} 
& & Non-infarcted & \multicolumn{2}{c}{ LAD infarct } \\
\cline { 3 - 5 } & & Pre-ablation & LBBB & Pre-ablation \\
\hline Radial displacement (mm) & Intra-obs. & $0.2 \pm 0.1$ & $0.2 \pm 0.1$ & $0.1 \pm 0.1$ \\
& Inter-seq. & $0.5 \pm 0.4$ & $0.3 \pm 0.3$ & $0.4 \pm 0.4$ \\
& Inter-obs. & $0.4 \pm 0.2$ & $0.4 \pm 0.3$ & $1.0 \pm 0.7$ \\
\hline Radial velocity (mm/s) & Intra-obs. & $1.9 \pm 1.5$ & $3.3 \pm 2.7$ & $1.5 \pm 1.5$ \\
& Inter-seq. & $7.0 \pm 6.5$ & $7.1 \pm 7.3$ & $4.5 \pm 4.2$ \\
& Inter-obs. & $4.6 \pm 4.1$ & $4.4 \pm 3.6$ & $8.0 \pm 6.3$ \\
\hline Circumferential strain (\%) & Intra-obs. & $2.2 \pm 1.1$ & $2.2 \pm 1.3$ & $0.8 \pm 0.6$ \\
& Inter-seq. & $3.7 \pm 3.7$ & $2.9 \pm 2.2$ & $0.3 \pm 0.2$ \\
& Inter-obs. & $3.8 \pm 2.9$ & $3.3 \pm 2.3$ & $5.0 \pm 3.9$ \\
\hline
\end{tabular}

LAD: left-anterior-descending; LBBB: left-bundle-branch-block. 


\section{Figure legends}

Figure 1: Pipeline for the comparison of motion and deformation patterns.

Figure 2: Effect of temporal alignment. Prevention of artifacts (black arrows) in pre/post-ablation radial velocity (median, non-infarcted animals).

Figure 3: Post-ablation changes on one non-infarcted animal. (a) ECG signals. (b) M-mode traces highlighting the presence of SF (yellow arrows).

Figure 4: Repeatability of the computations illustrated for one non-infarcted animal.

Figure 5: Difficulty of characterizing dyssynchrony by single measurements (peak identification), on one noninfarcted animal.

Figure 6: Radial displacement, velocity and circumferential strain for each subgroup. Septal/lateral curves (median and first/third quartiles) and full spatiotemporal (median) maps. Black arrows highlight the presence of SF, double-peaked systolic septal shortening, early-systole lateral wall stretch and post-systolic abnormal motion. 
Figure1

Click here to download Figure: Fig1.eps

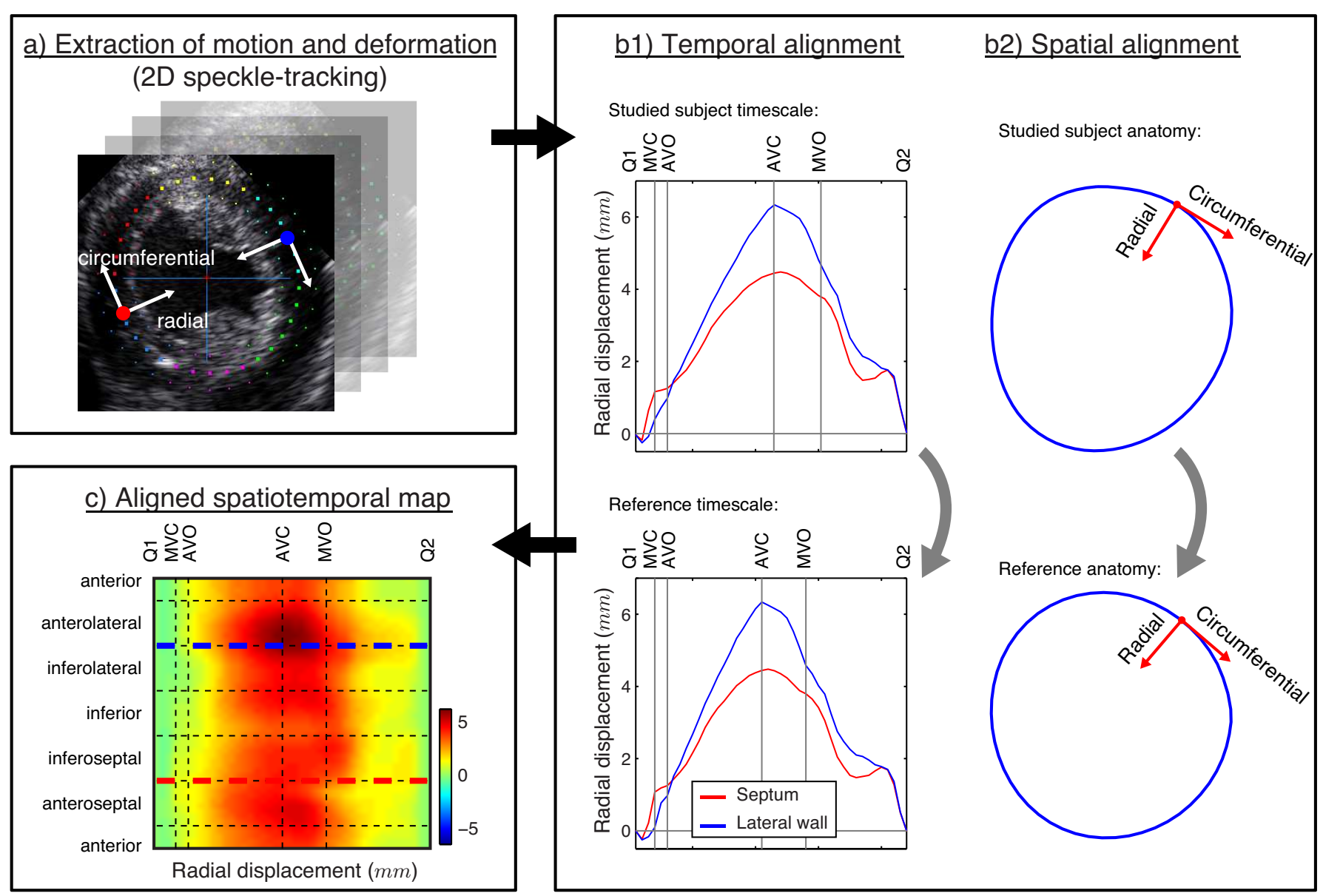

Q1, Q2: onset of QRS; AVO/AVC/MVO/MVC: aortic/mitral valve opening/closure. 

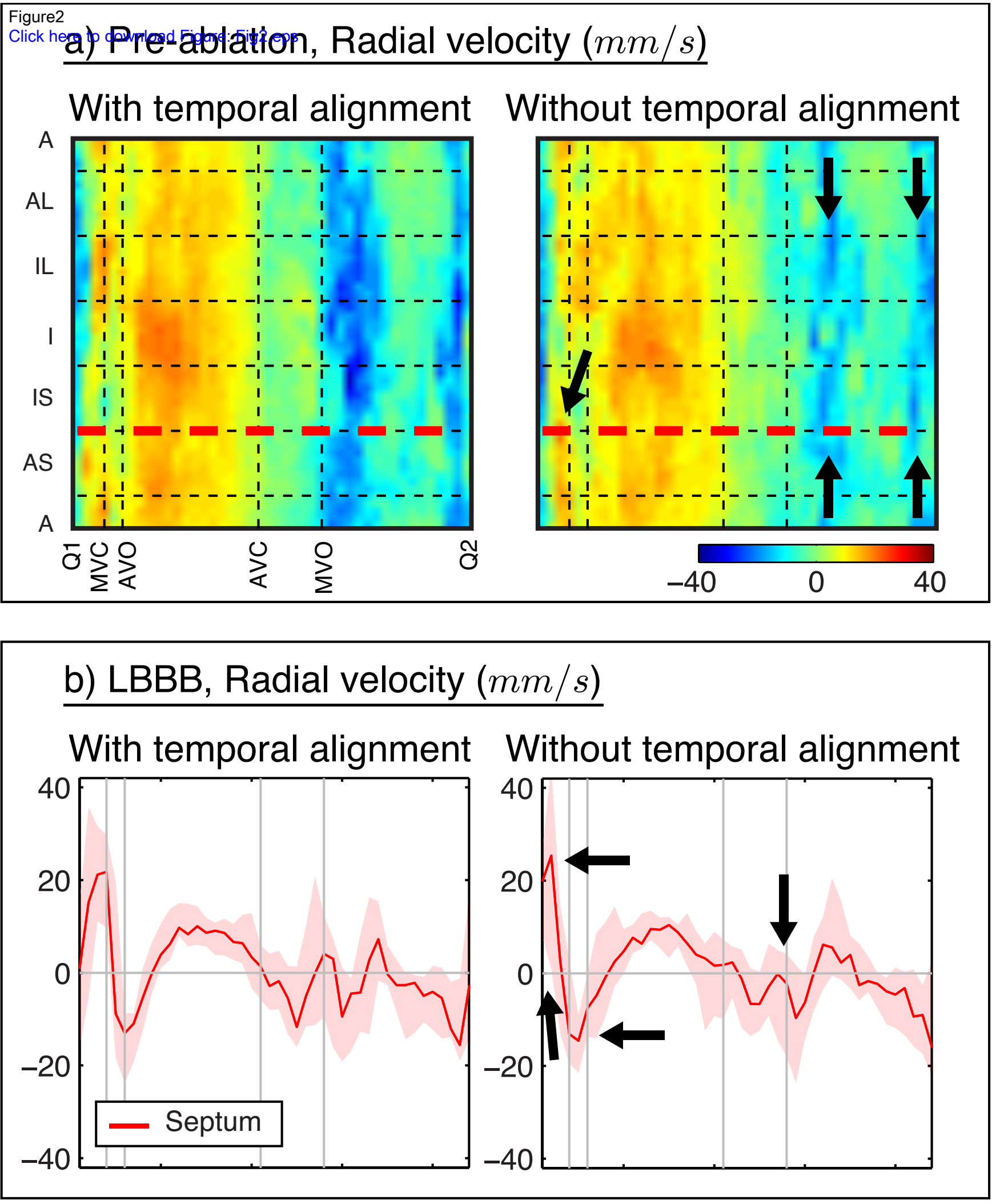

Q1, Q2: onset of QRS; AVO/AVC/MVO/MVC: aortic/mitral valve opening/closure.

A: anterior; AL: anterolateral; IL: inferolateral; I: inferior; IS: inferoseptal; AS: anteroseptal. LBBB: left-bundle-branch-block. 
Preprint version accepted to appear in International Journal of Cardiovascular Imaging.

Final version of this paper available at http://link.springer.com/article/10.1007\%2Fs10554-014-0403-2

\section{Figure3}

Click here to download Figure: Fig3.eps

(a) ECG signals

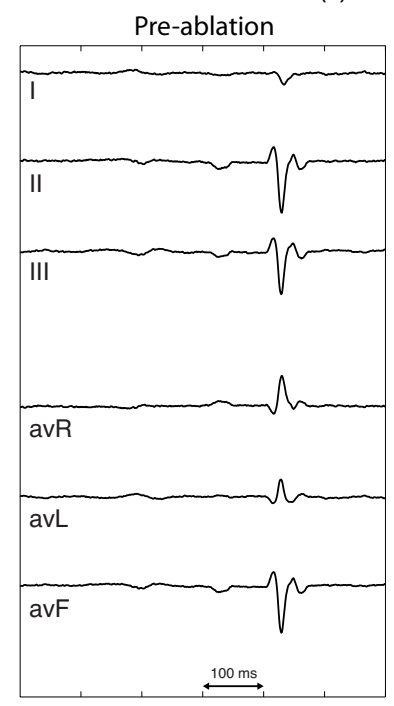

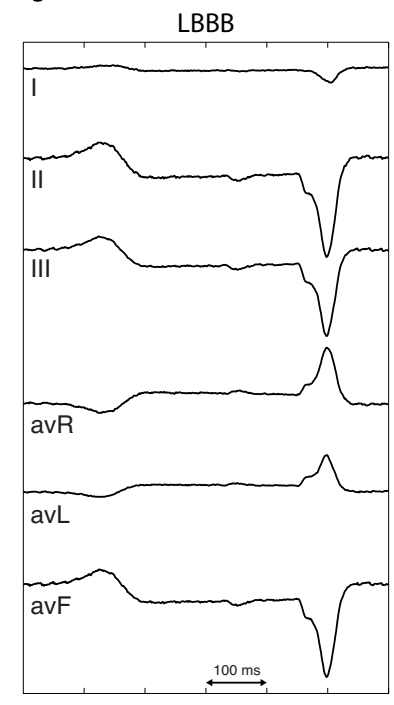

(b) Mmode traces

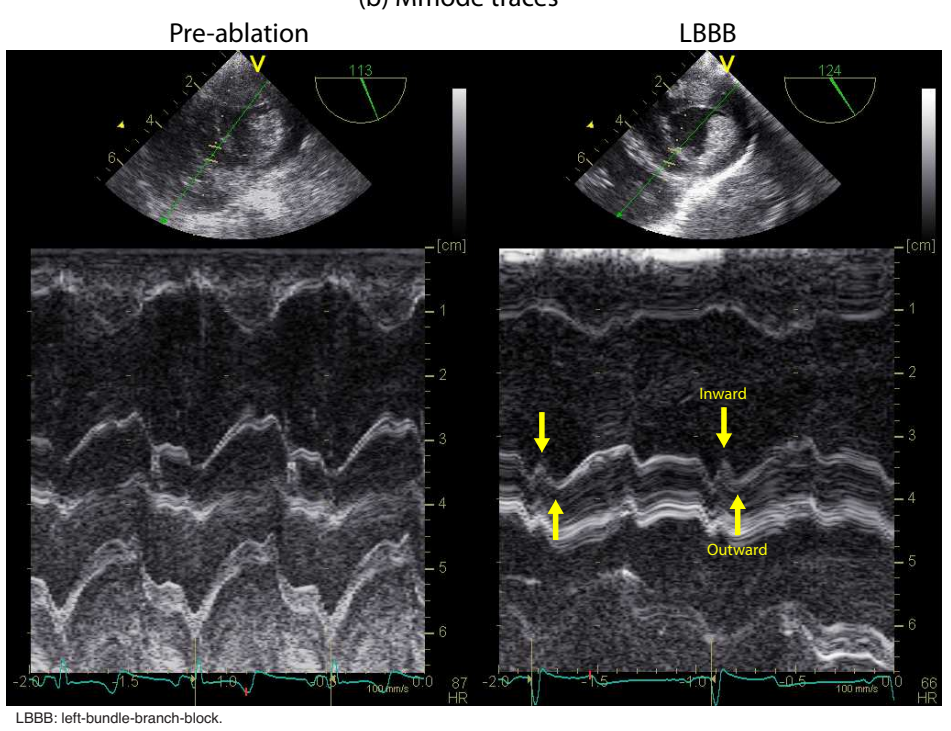


Figure4

Click here to download Figure: Fig4.eps
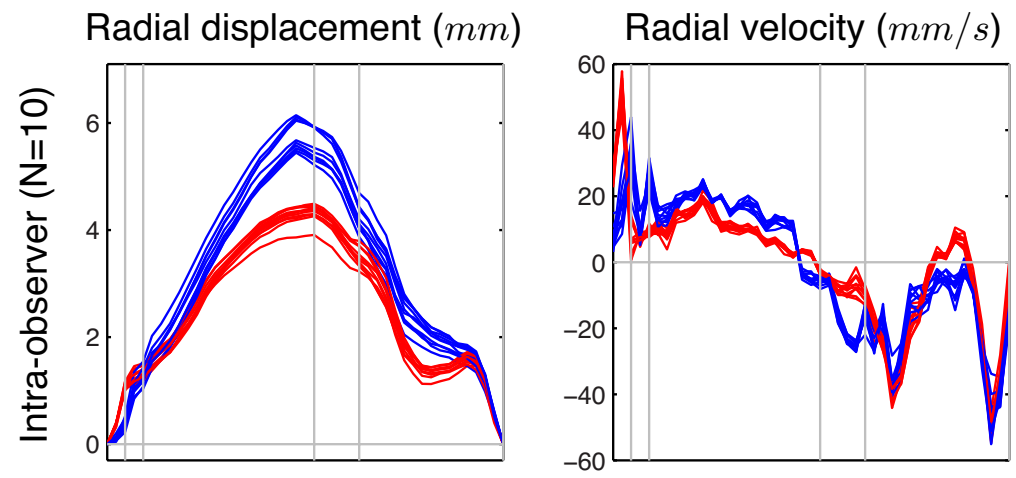

Circumferential strain (\%)
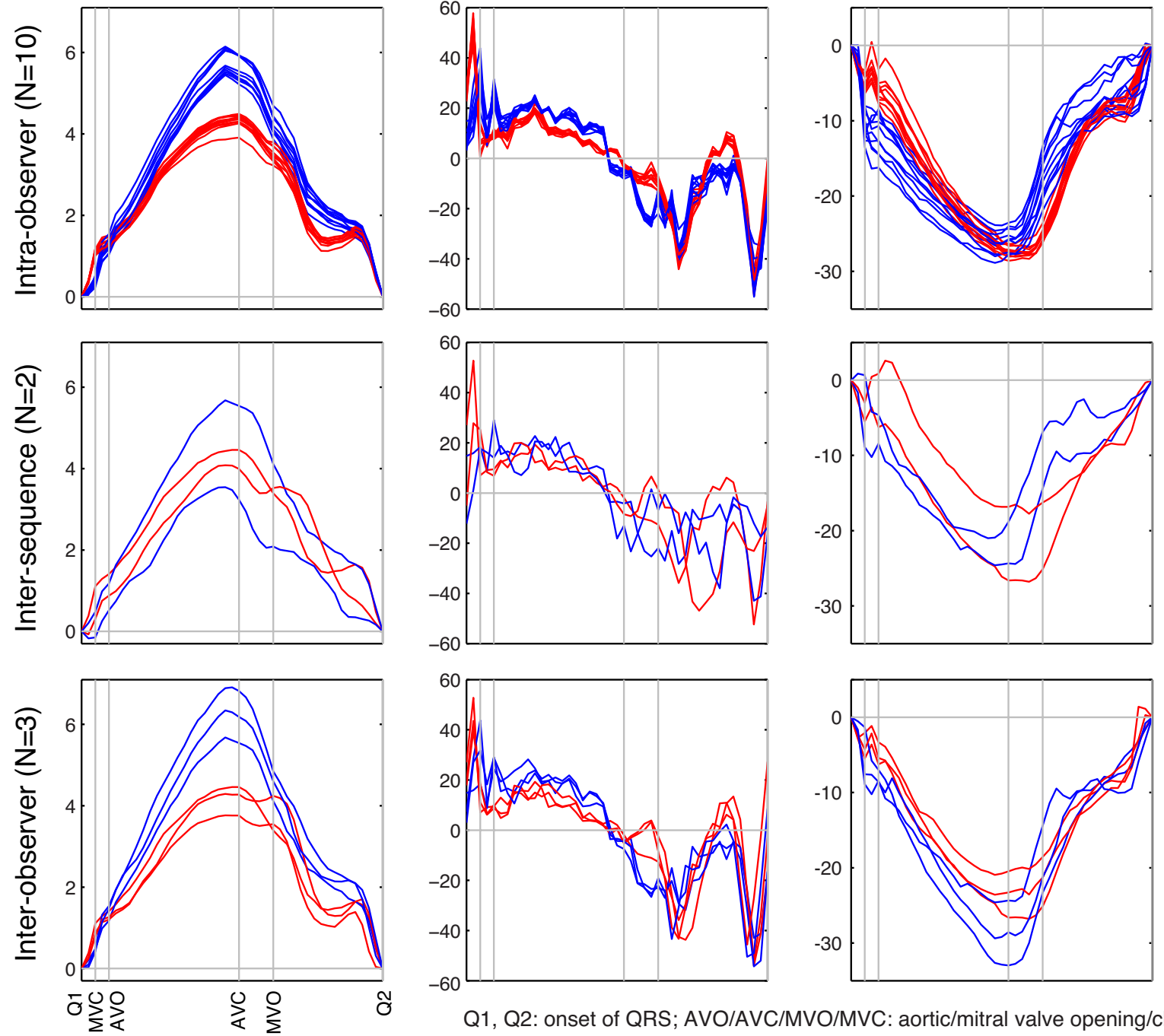

- Septum
— Lateral wall

Q1, Q2: onset of QRS; AVO/AVC/MVO/MVC: aortic/mitral valve opening/closure. 
Circumferential strain (\%)

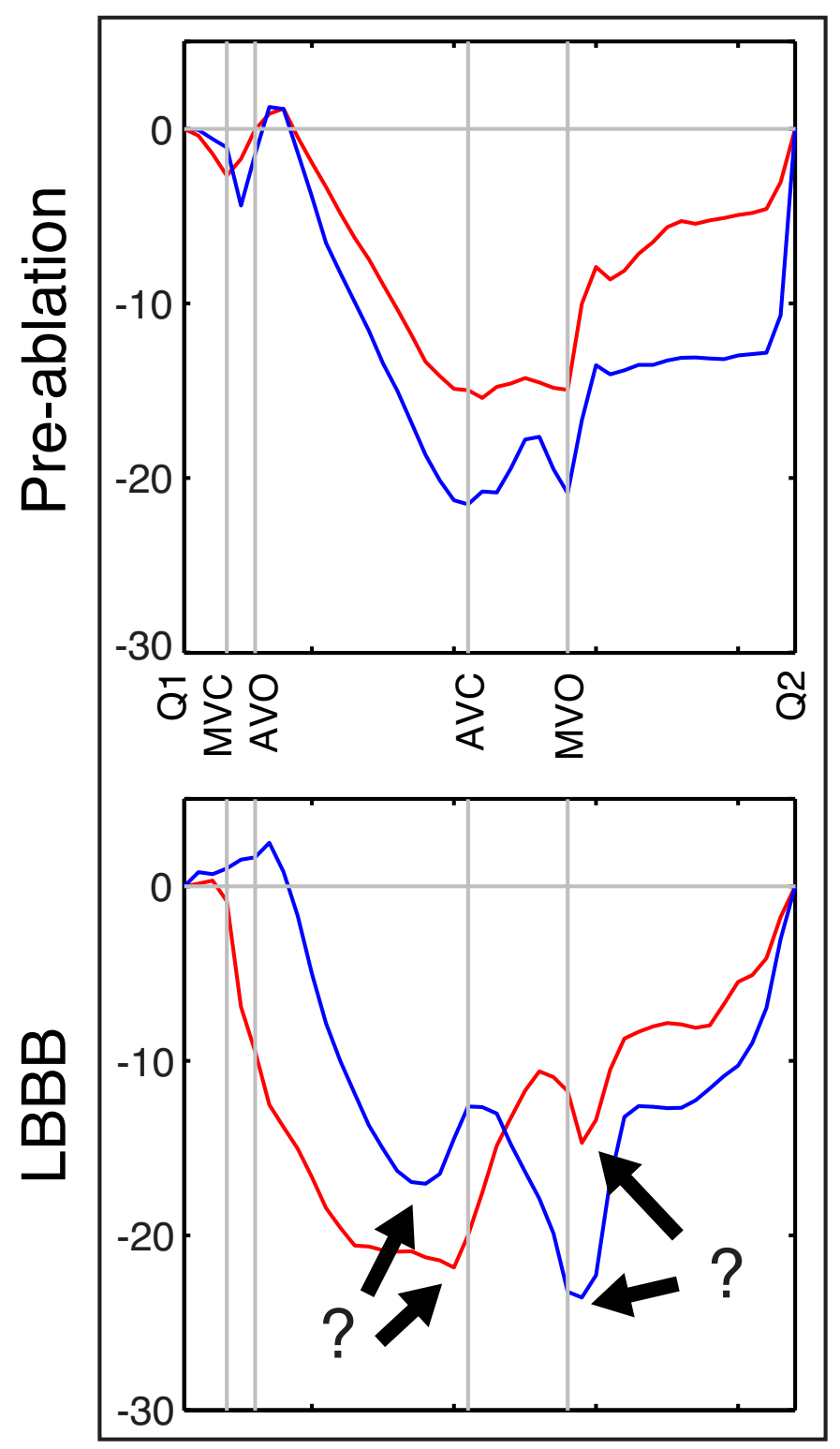

Radial displacement $(\mathrm{mm})$

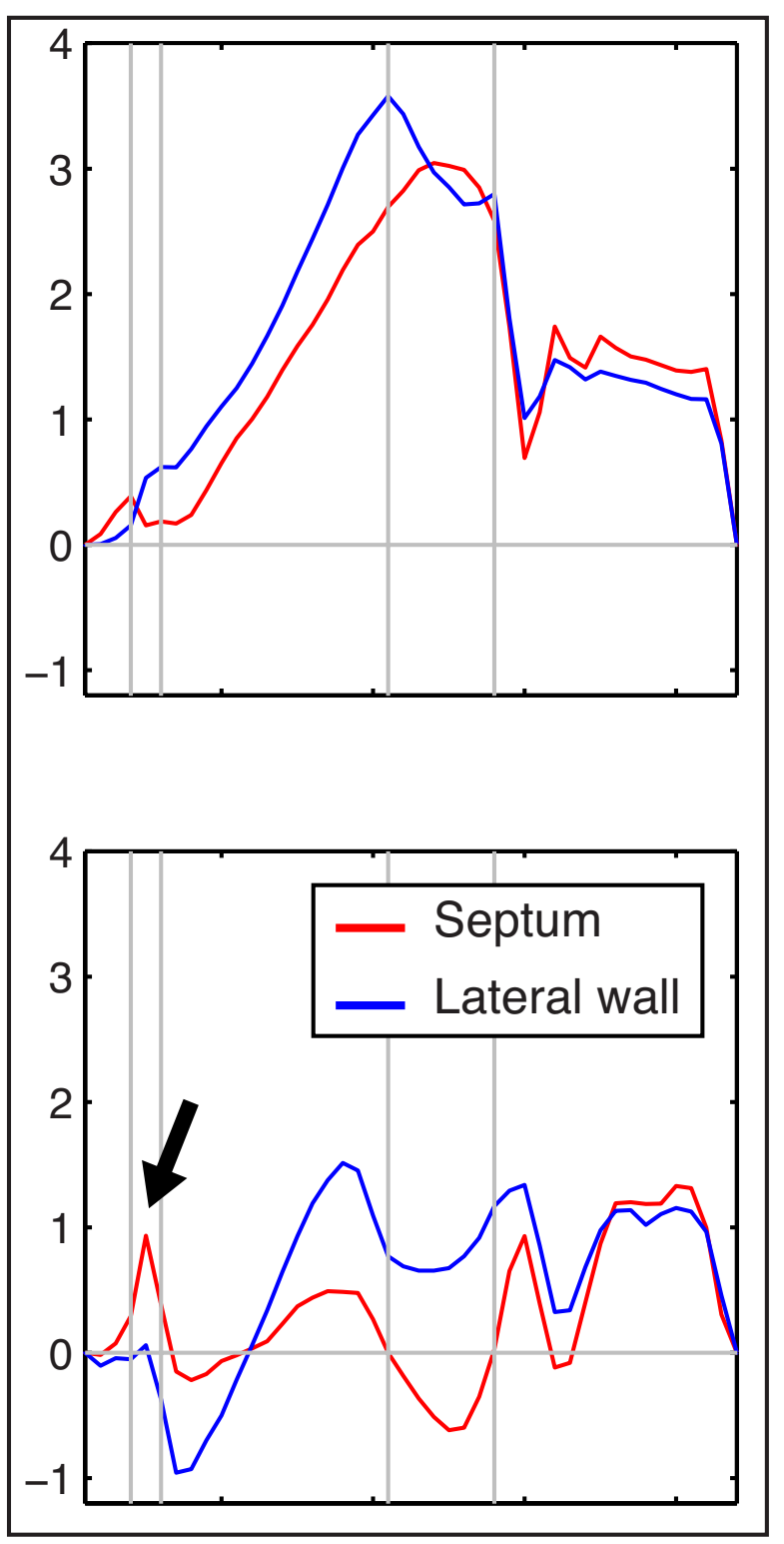

Q1, Q2: onset of QRS; AVO/AVC/MVO/MVC: aortic/mitral valve opening/closure. LBBB: left-bundle-branch-block. 

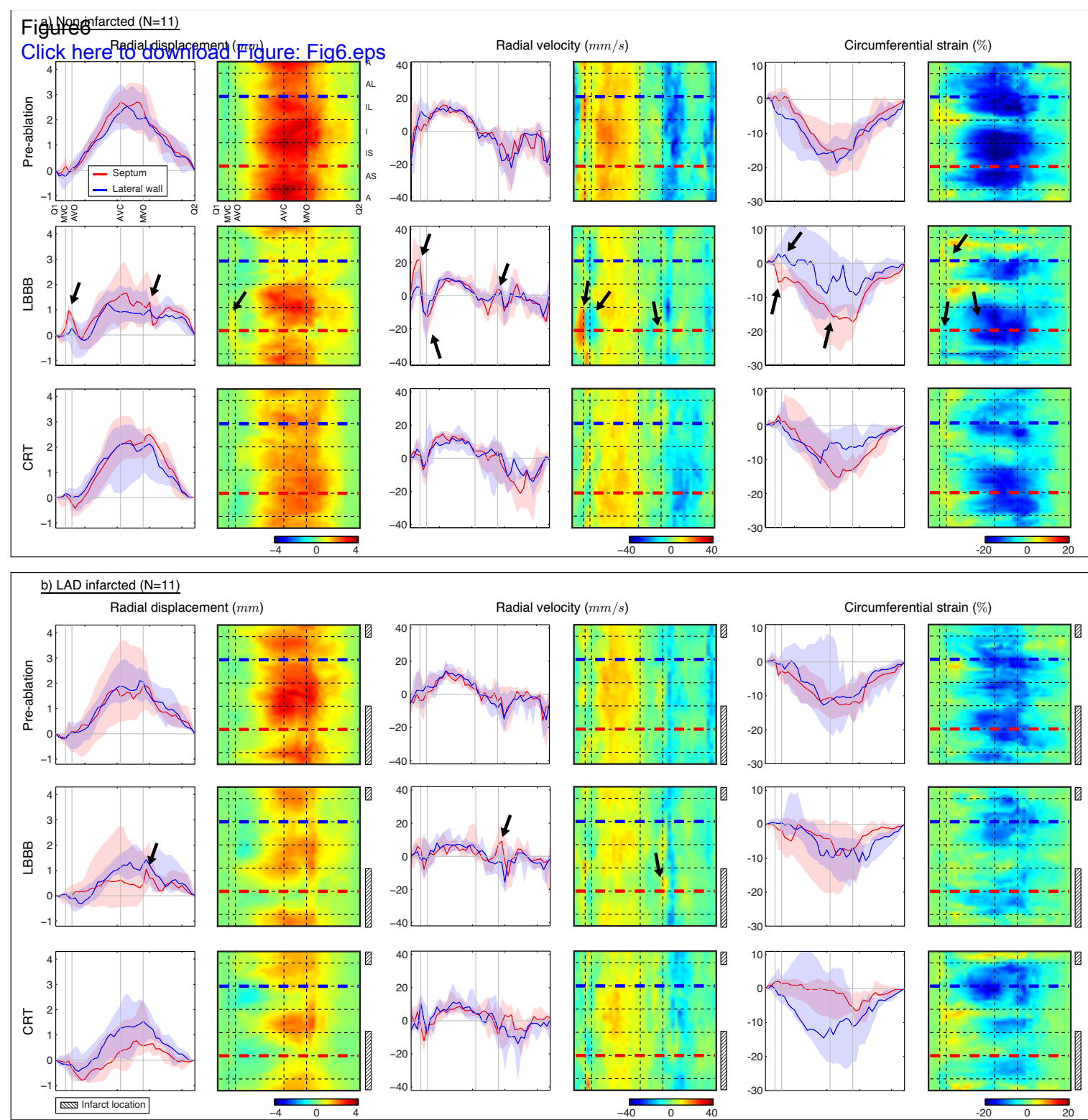

Q1, Q2: onset of QRS; AVO/AVC/MVO/MVC: aortic/mitral valve opening/losure.

A: anterior; AL: anterolateral; IL: inferolatereal; i: inferior; ;S: inferoseptal; AS: anteroseptal.
LBBB: lett-bundle-branch-block; CRT: cardiac resynchronization therapy: LAD: left-anterior-descending. 